\title{
SPRY2 is a novel MET interactor that regulates metastatic potential and differentiation in rhabdomyosarcoma
}

\author{
Masum Saini ${ }^{1}$, Aakanksha Verma ${ }^{1}$ and Sam J. Mathew ${ }^{1}$
}

\begin{abstract}
Rhabdomyosarcoma (RMS) is a predominantly pediatric soft-tissue cancer where the tumor cells exhibit characteristics of the developing skeletal muscle, and the two most common sub-types are embryonal and alveolar RMS. Elevated activation of the receptor tyrosine kinase (RTK) MET is frequent in RMS and is thought to cause increased tumor metastasis and lack of differentiation. However, the reasons underlying dysregulated MET expression and activation in RMS are not well understood. Therefore, we explored the role of Sprouty 2 (SPRY2), a modulator of RTK signaling, in regulating MET. We identify SPRY2 as a novel MET interactor that colocalizes with and binds MET in both embryonal and alveolar RMS. We find that depletion of SPRY2 leads to MET degradation, resulting in reduced migratory and clonogenic potential, and induction of differentiation in both embryonal and alveolar RMS, outcomes that are identical to depletion of MET. Activation of the ERK/MAPK pathway, known to be crucial for regulating cell migration and whose inhibition is required for myogenic differentiation, was downregulated upon depletion of MET or SPRY2. This provides a direct connection to the decreased migration and induction of differentiation upon depletion of MET or SPRY2. Thus, these data indicate that SPRY2 interacts with MET and stabilizes it in order to maintain signaling downstream of MET, which keeps the ERK/MAPK pathway active, resulting in metastatic potential and inhibition of differentiation in RMS. Our results identify a novel mechanism by which MET signaling is stabilized in RMS, and is a potential target for therapeutic intervention in RMS.
\end{abstract}

\section{Introduction}

Rhabdomyosarcoma (RMS) is the most common pediatric soft-tissue sarcoma, accounting for about $3 \%$ of childhood cancers ${ }^{1}$. It is a relatively rare $(\sim 4.5$ cases per million children annually), but aggressive malignancy ${ }^{2-4}$. The most common variants are embryonal (ERMS; 67\%) and alveolar rhabdomyosarcoma (ARMS; $30 \%$ ), which exhibit distinct clinical and molecular features ${ }^{5,6}$. Histopathologically, ERMS tumors are characterized by zones of hypo and hyper-cellularity, whereas loose nests of rounded cells interspersed by fibro-vascular septa are

\footnotetext{
Correspondence: Sam J. Mathew (sjmathew@rcb.res.in)

${ }^{1}$ Laboratory of Developmental Genetics, Regional Centre for Biotechnology, NCR Biotech Science Cluster, 3rd Milestone, Faridabad-Gurgaon Expressway, Faridabad, Haryana 121001, India

Edited by G. Melino
}

characteristic of ARMS ${ }^{7}$. ARMS is highly aggressive, frequently characterized by the chromosomal translocations $\mathrm{t}(2 ; 13)$ involving $P A X 3-F K H R$, or $\mathrm{t}(1 ; 13)$ involving $P A X 7$ $F K H R$ fusion. ERMS has a relatively more favorable prognosis, and is associated with loss of heterozygosity of 11p15.5, p53 pathway disruption and RAS activation ${ }^{8}$.

RMS tumors show morphological similarities to developing muscle cells and express muscle differentiation markers such as MyoD, myogenin, and myosin heavy chain $(\mathrm{MHC})^{4,9-12}$. Thus, RMS tumor cells recapitulate the embryonic myogenic program, although unlike embryonic myogenesis where cells exit the proliferative cycle upon terminal differentiation, the tumor cells persist in an undifferentiated state. Despite their resemblance to myogenic cells, the cell type of origin in RMS is debated. RMS have been proposed to arise from skeletal muscle 


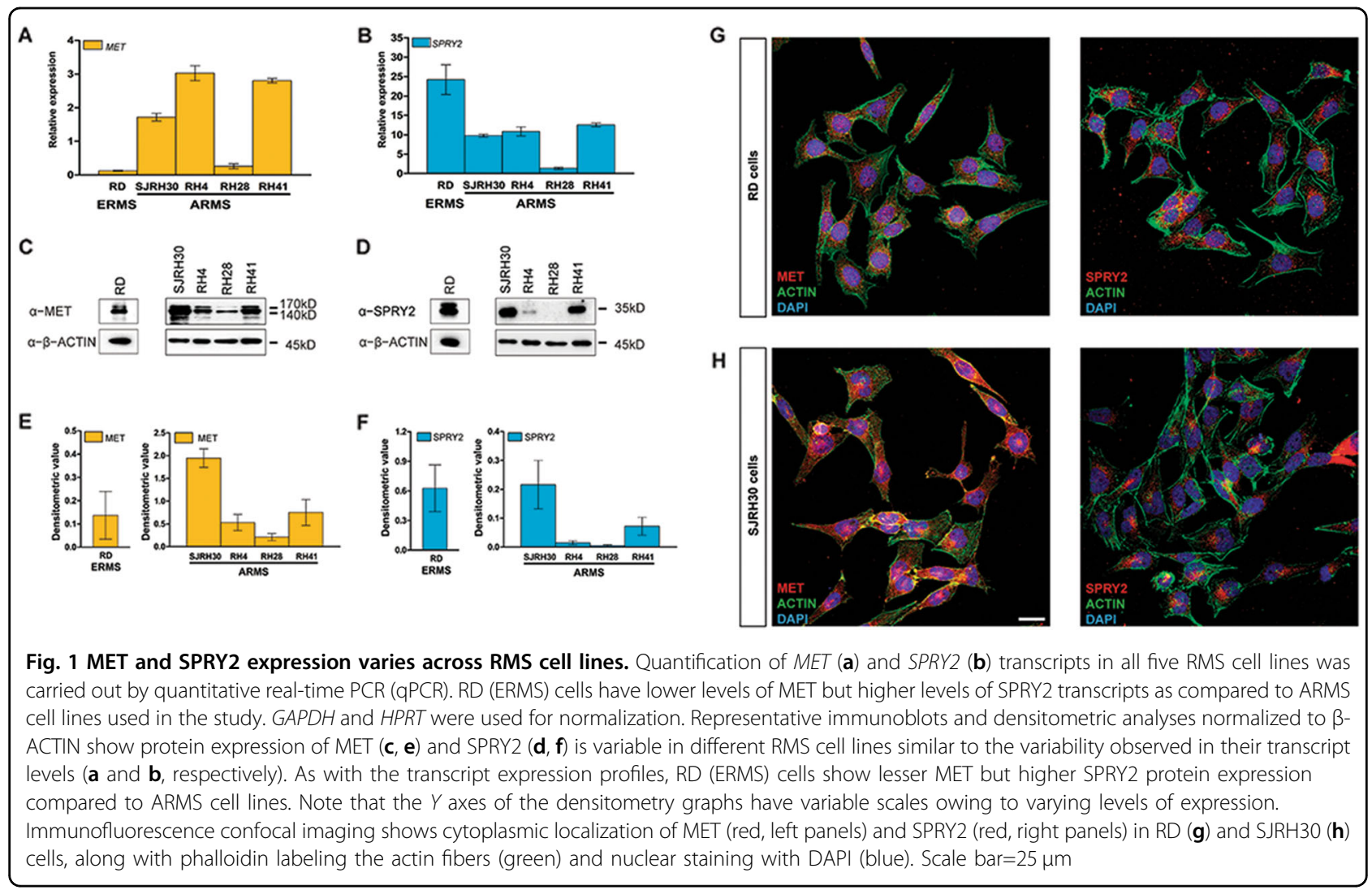

stem cells (satellite cells), de-differentiation of terminally differentiated myogenic cells, or mesenchymal stem cells committing to the skeletal muscle lineage ${ }^{13-15}$.

Another common thread between mammalian myogenesis and RMS tumors is the expression of a receptor tyrosine kinase (RTK)-MET, by the myogenic progenitors and RMS cells ${ }^{16-19}$. MET was identified as a fusion oncogene in osteosarcoma, and is known to control cell proliferation, survival, and migration, in response to binding by its ligand hepatocyte growth factor (HGF) during developmental morphogenesis and in multiple cancer types ${ }^{20,21}$. During mammalian development, MET expression in myogenic precursors is required for their migration to target organs such as limbs ${ }^{16,17}$. During adult regenerative myogenesis, MET activates and regulates satellite cell migration, and controls myocyte fusion ${ }^{22-24}$. Interestingly, MET is overexpressed, aberrantly activated, essential for metastasis and inhibition of differentiation in RMS, and is a potential candidate for therapeutic targeting $^{18,19,25-27}$. Thus, identification of MET regulators will be critical to understanding RMS pathology, and attenuating MET signaling by targeting MET or its regulators, could serve as intervention points in RMS patients.

Regulation of RTK signaling cascades is essential for physiological homeostasis ${ }^{28}$. The Sprouty (SPRY) family of proteins are important modulators of RTK signaling and SPRY2, a member of the family, functions as a bimodal regulator ${ }^{29,30}$. Versatility of SPRY2 in modulating RTK-mediated signaling is cell type, and RTK context dependent, which can result in opposing effects, potentiating or dampening signals transduced from RTKs ${ }^{30,31}$. While SPRY2 inhibits fibroblast growth factor (FGF)mediated extracellular-signal-regulated kinase (ERK) signaling by preventing RAF activation, it augments epidermal growth factor receptor (EGFR)-induced ERK signaling, by inhibiting EGFR endocytosis and degradation $^{32,33}$. SPRY2 also exhibits contrasting tumor suppressive or oncogenic roles in different cancer contexts $^{34-36}$. For example, overexpression of SPRY2 negatively regulated HGF-mediated ERK and AKT signaling in human leiomyosarcoma, whereas SPRY2 overexpression increased MET activation resulting in enhanced cell migration and invasion in colonic adenocarcinomas ${ }^{35,36}$.

Association of MET activity with enhanced metastatic potential and inhibition of differentiation underscores the importance of understanding MET regulation in RMS. Since regulation of MET in RMS is largely unexplored and reports indicate that SPRY2 can alter MET signaling in cancers, we carefully analyzed MET, SPRY2 and their role in RMS, using representative RMS cell lines. Loss of SPRY2 function led to a significant reduction in MET protein levels in RMS cells, mediated primarily by the 


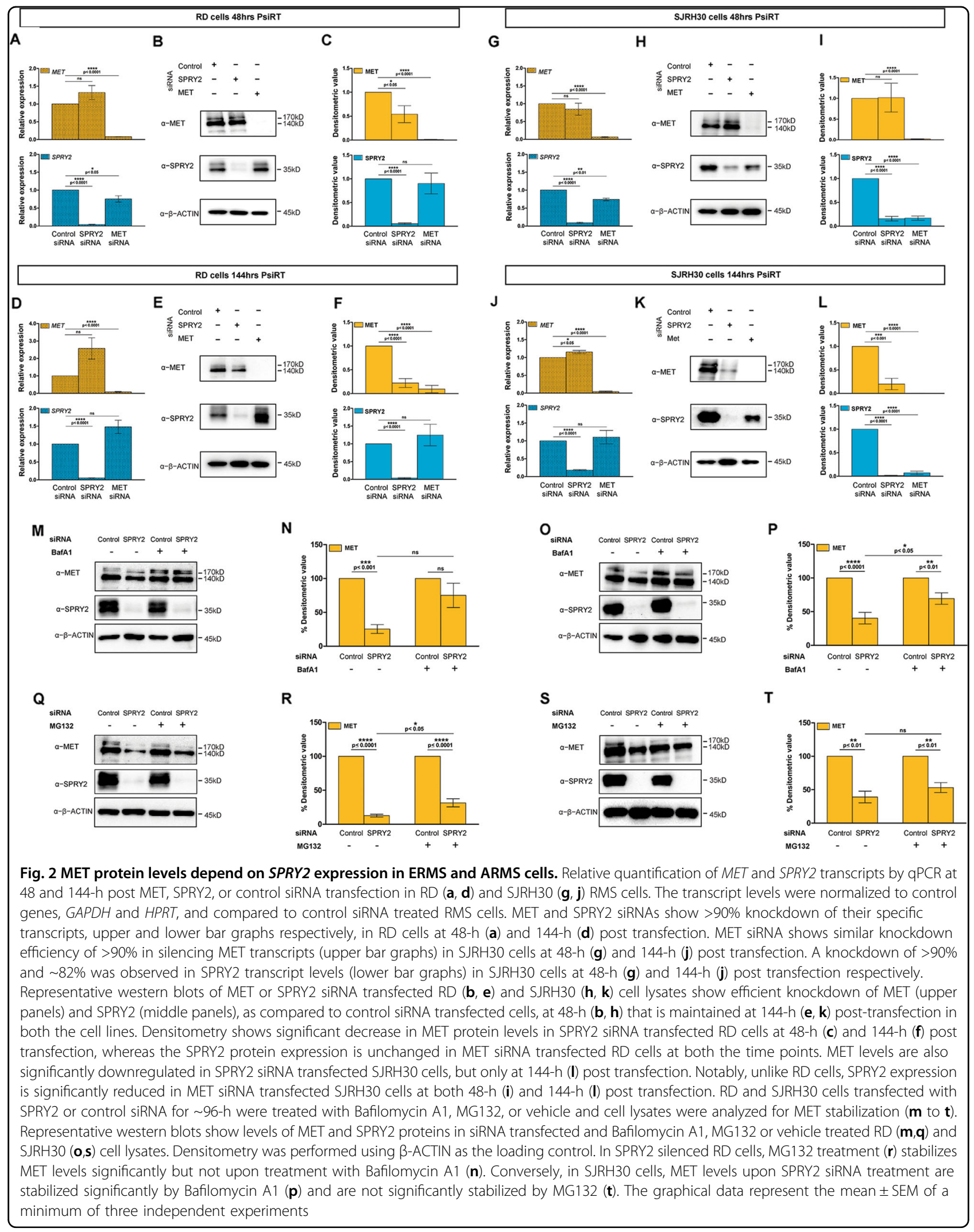


proteasomal pathway in ERMS and lysosomal pathway in ARMS. We uncovered that MET and SPRY2 interact physically and colocalize with each other in RMS cells. Notably, knockdown of SPRY2 or MET lead to similar functional outcomes in RMS cells, mediated by reduced ERK activation resulting in decreased migratory potential, and inducing differentiation. Thus, our study shows that SPRY2 is a key interactor and regulator of MET in RMS, functioning to stabilize the MET receptor to sustain downstream signaling, which is essential for maintenance of migratory, metastatic and clonogenic capabilities, as well as to inhibit differentiation in RMS.

\section{Results}

MET and SPRY2 expression varies between RMS cell lines

MET is upregulated in RMS ${ }^{18,19}$, and SPRY2 has been reported to regulate RTK signaling ${ }^{32,33}$. No reports exist on SPRY2 as a regulator of MET signaling in RMS. Therefore, to ascertain whether SPRY2 has any role in regulating MET in RMS, we characterized SPRY2 and MET expression in embryonal (RD, representative ERMS cell line) and alveolar (SJRH30, RH4, RH28, RH41) RMS cell lines. We found that MET transcript (Fig. 1a) and protein (Fig. 1c, e) expression was higher in ARMS compared to ERMS cells, whereas SPRY2 transcript (Fig. 1b) and protein (Fig. 1d, f) expression was higher in ERMS than ARMS cells. Expression of MET and SPRY2 proteins varied across the different RMS cell lines, but broadly in ARMS, cell lines with higher MET levels also exhibited elevated SPRY2 (Fig. 1c-f). MET and SPRY2 exhibited cytoplasmic localization in RD and SJRH30 cells (Fig. 1g, h). Among the ARMS cell lines, SJRH30 showed highest levels of MET and SPRY2 (Fig. 1e, f) and was chosen as the representative ARMS cell line, along with RD cells (ERMS), to investigate the regulation of MET signaling by SPRY2.

\section{SPRY2 regulates levels of MET receptor in RMS}

Multiple reports in different cancer types indicate that SPRY2 regulates MET levels ${ }^{34-36}$. The MET ligand HGF, and ERK signaling, a downstream effector of RTKs, are known to induce SPRY2 expression ${ }^{35,37}$. However, no studies have investigated MET, SPRY2 and their interactions in RMS. Since we observed elevated MET and SPRY2 levels, as well as similar cytoplasmic localization of both, we examined whether they exert regulatory effects on each other by knocking them down individually using small interfering RNA (siRNA) in RD and SJRH30 cells (Fig. 2). We validated the knockdown at the transcript (Fig. 2a, d, g, j) and protein (Fig. 2c, f, i, l) levels and observed $>80 \%$ knockdown efficiency at early $(48-\mathrm{h})$ and late (144-h) time points post-siRNA transfection (PsiRT).

Upon MET knockdown, SPRY2 transcript levels decreased significantly at 48-h PsiRT in RD and SJRH30
(Fig. 2a, g, lower graphs) cells, indicating that MET signaling regulates SPRY2 transcriptional activation, as reported in leiomyosarcoma ${ }^{35}$. However, this effect was not seen 144-h PsiRT, suggesting that compensatory signals restore SPRY2 transcriptional activation (Fig. 2d, j, lower graphs). At the protein level upon MET knockdown, SPRY2 levels were unchanged in RD cells (Fig. 2b, e, middle panels and C, F, lower graphs), whereas it was significantly downregulated in SJRH30 cells at 48 and 144-h (Fig. 2h, k, middle panels and I, L, lower graphs). This suggests intrinsic differences between ERMS and ARMS, and is likely due to enhanced MET expression in ARMS compared to ERMS ${ }^{38}$.

Upon SPRY2 knockdown, we did not observe any effects on MET transcript levels in RD (Fig. 2a, d, upper graphs), or SJRH30 cells at 48-h (Fig. 2g, upper graph), but a subtle upregulation was apparent at 144-h (Fig. 2j, upper graph). At the protein level, SPRY2 knockdown resulted in a striking reduction in MET by 144-h in RD and SJRH30 cells, indicating that SPRY2 depletion leads to MET degradation (Fig. 2e, f, k, 1 upper panels). These results were validated using a separate set of siRNAs targeting MET and SPRY2 (Supplementary Figure 1). Reduction in MET protein following SPRY2 depletion cannot be transcriptional, since MET transcript levels were not reduced upon SPRY2 knockdown (Fig. 2a, d, g, j upper graphs). Our findings suggest that MET partly regulates SPRY2 transcriptional activation in RMS, and SPRY2 protein levels in ARMS. On the other hand, SPRY2 has negligible effect on MET transcript levels, but is required to maintain $\mathrm{MET}$ protein levels, indicating that SPRY2 might be a crucial factor regulating MET in RMS.

Therefore, we tested whether MET undergoes degradation when SPRY2 is silenced in RMS cells. MET is degraded by proteasomal and lysosomal pathways ${ }^{39}$. Upon SPRY2 depletion, proteasomal inhibition with MG132 prevented MET degradation in RD cells (Fig. 2q, $\mathrm{r}$ ), and although not statistically significant, a trend towards MET stabilization upon Bafilomycin A1mediated lysosomal inhibition was observed (Fig. 2m, n). Conversely, MET levels stabilized significantly upon Bafilomycin A1 treatment in SPRY2 silenced SJRH30 cells (Fig. 2o, p), whereas MET stabilization upon proteasomal inhibition was insignificant in these cells (Fig. 2s, t). These findings indicate that SPRY2 regulates MET levels by preventing its degradation, through distinct pathways in the two RMS sub-types.

\section{SPRY2 colocalizes and interacts with MET in RMS}

Since SPRY2 regulates MET and silencing SPRY2 decreased MET protein levels, we investigated whether SPRY2 interaction with MET is required for stabilizing and preventing MET degradation in RMS. To test this, we labeled MET and SPRY2 proteins by 


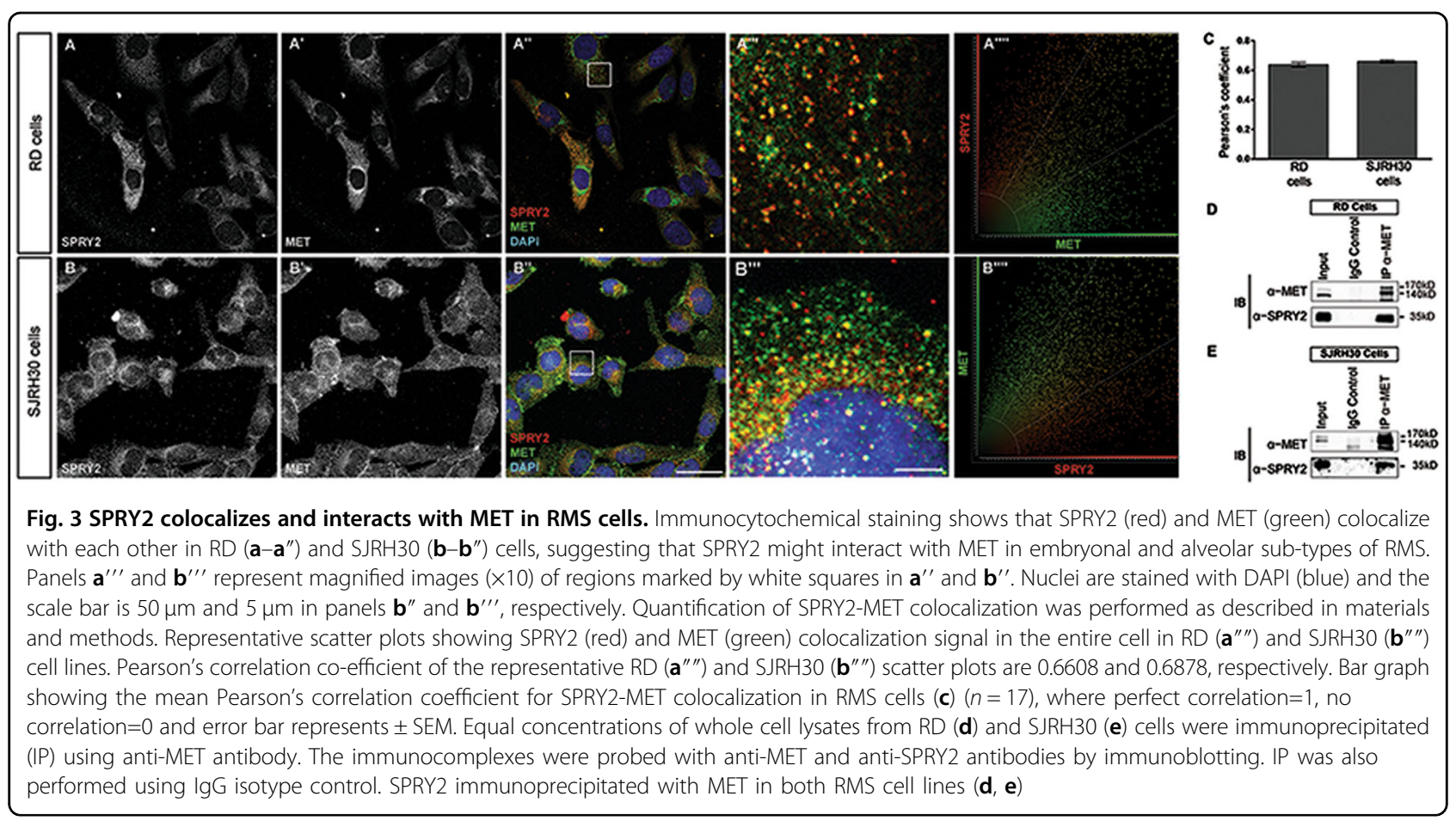

immunocytochemistry and both proteins localized to cytoplasmic punctae, colocalizing with each other as measured by Pearson's correlation coefficient in RD and SJRH30 cells (Fig. 3a-a"', b- b"'", c) ${ }^{40}$. To validate whether colocalization was an indication of biochemical interaction, we probed MET immunoprecipitates from RMS cell lysates with SPRY2 antibody. Interestingly, SPRY2 immunoprecipitated with MET in RD and SJRH30 cells (Fig. 3d, e). To our knowledge, no previous studies have shown that effects of SPRY2 on RTK regulation are mediated by a physical interaction between SPRY2 and the RTK. Thus, our findings indicate that SPRY2 and MET interact, which possibly is crucial to MET stability.

MET regulates cell proliferation and survival during development and in different cancers ${ }^{20}$. Since we found that SPRY2 regulates MET levels, we examined whether silencing SPRY2 or MET leads to altered cell death and proliferation in RMS. Evaluation of Cyclin D1 levels in MET depleted RD or SJRH30 cells (Supplementary Figure $2 \mathrm{a}, \mathrm{b}$ and $\mathrm{e}, \mathrm{f}$ ) indicate that cell proliferation is not significantly impaired, suggesting that additional pathways regulate proliferation in RMS. However, Cyclin D1 levels were reduced significantly in SPRY2 depleted RD but not SJRH30 cells (Supplementary Figure 2a, b and e, f), indicating that SPRY2 may modulate pathways controlling cell proliferation in ERMS, which has higher SPRY2 levels, differently than in ARMS. Cleaved Caspase3 measuring cell death showed a slight, statistically insignificant increase in RD cells and a significant decrease in SJRH30 cells upon SPRY2 depletion (Supplementary Figure 2c, d and g, h). Cell death was significantly increased upon MET depletion in RD but was unaffected in SJRH30 cells (Supplementary Figure 2c, d and g, h). Overall, MET or SPRY2 depletion affected cell death and proliferation differently in ERMS and ARMS, highlighting the intrinsic differences between the RMS sub-types.

\section{SPRY2 and MET are required to increase migratory and clonogenic potential and inhibit differentiation in RMS}

Since SPRY2 interacts with and stabilizes MET, we studied the effect of SPRY2 or MET silencing on RMS cell migration, clonogenic potential and differentiation. We assessed the migratory potential of SPRY2 or MET depleted RD and SJRH30 cells by wound healing assay ${ }^{41}$. As compared to control (Fig. 4a, top panel), MET (Fig. 4a, bottom panel) or SPRY2 (Fig. 4a, middle panel) depleted $\mathrm{RD}$ cells showed significantly reduced percentage of wound closure (Fig. 4b) over the assay duration. Similarly, cell motility was significantly compromised in SPRY2 or MET depleted SJRH30 cells (Fig. 4d). Thus, MET (Fig. 4a, c, bottom panels) or SPRY2 (Fig. 4a, c, middle panels) silenced RD and SJRH30 cells failed to close the wound 42 and 24-h respectively after scratching the monolayer (Fig. 4a, c, top panels). End point immunoblotting validated that SPRY2 and MET remained silenced during the assay (Fig. 4b', d'). Hence, depleting SPRY2 impaired motility in ERMS and ARMS cells (Fig. 4), mimicking the 

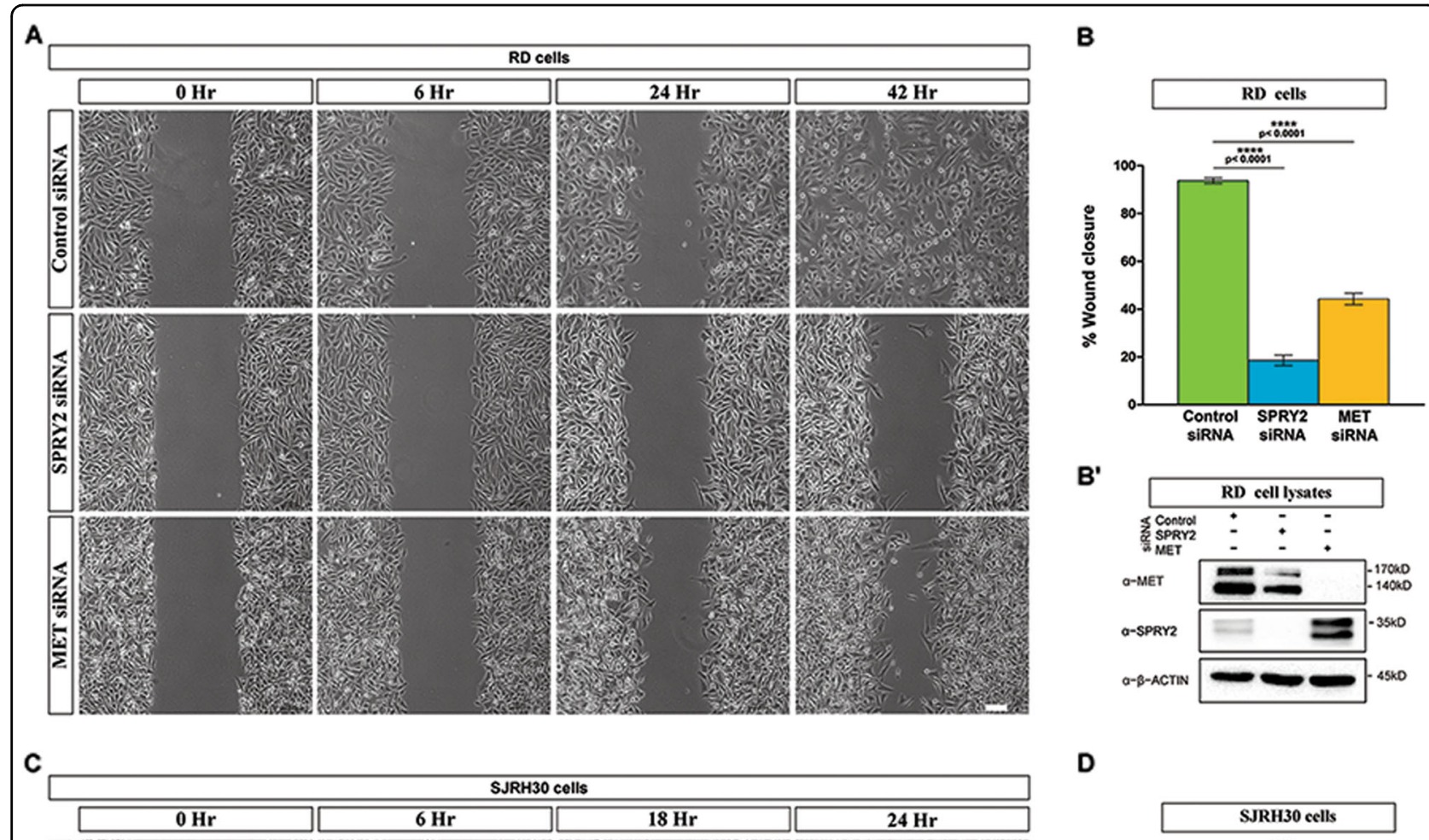

\section{D}

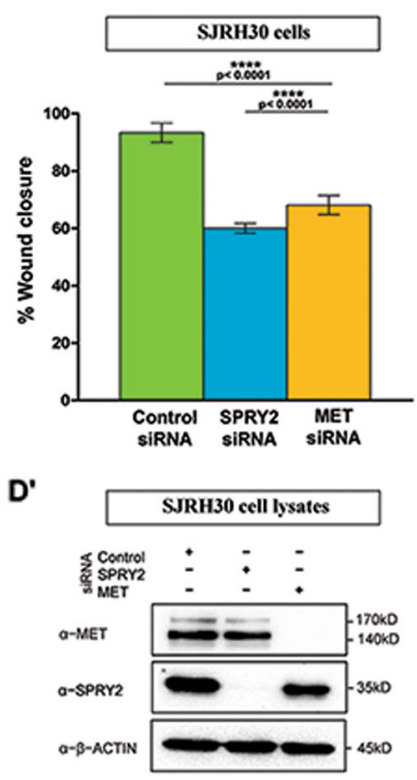

Fig. 4 Knockdown of SPRY2 significantly reduces RMS cell migratory potential recapitulating the effect of MET downregulation.

Representative bright field micrographs of wound closure assays on RD (a) and SJRH30 (c) RMS cells imaged at specific time points after 96-h of transfection with MET or SPRY2 siRNAs, compared to those transfected with control siRNA. Scale bar is $100 \mu \mathrm{m}$. Bar graphs summarizing percent wound closure in the monolayers of RD (b) and SJRH30 (d) cells indicates that wound closure is significantly decreased in MET and SPRY2 siRNA transfected RMS cells when compared to control siRNA transfected cells. The graphical data represent the mean \pm SEM of a minimum of 5 independent experiments. Representative western blots using cell lysates prepared at the end of the scratch assay show that MET (upper panels) and

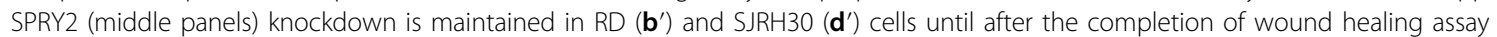

effect of MET knockdown. We validated these findings using a transwell migration assay where the percentage migration in SPRY2 or MET depleted RD (Fig. 5a, b) and SJRH30 (Fig. 5g, h) cells was significantly impaired.
Next, we assessed the effect of silencing SPRY2 or MET on the clonogenic potential of RMS cells by colony forming assays. Silencing SPRY2 or MET significantly inhibited attachment-independent clonal growth in RD cells (Fig. 5c, d) but not in SJRH30 cells (Fig. 5i, j). This 


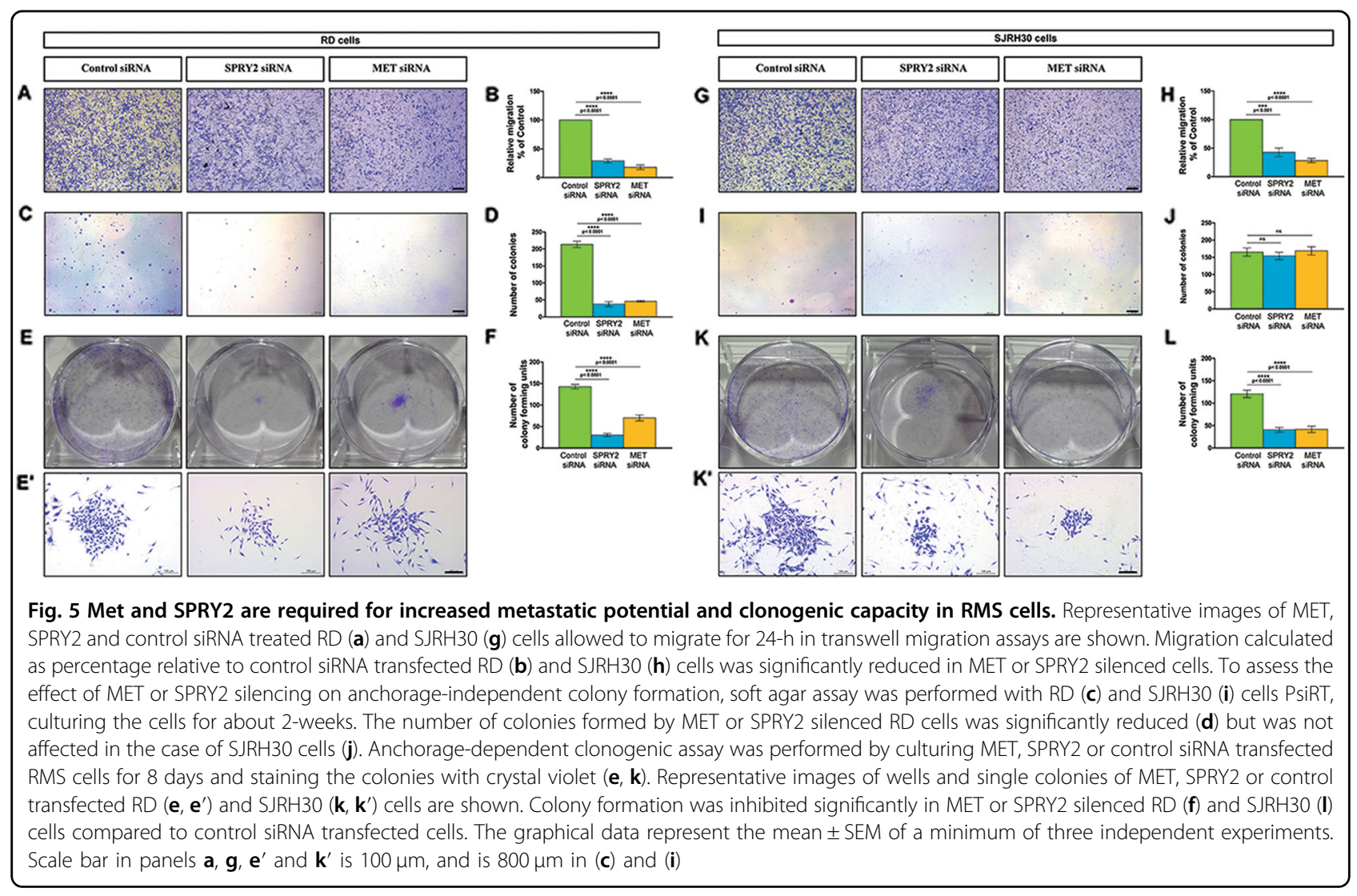

disparity between the RMS sub-types may be due to their differential levels of MET, and inability of siRNAmediated knockdown to maintain MET downregulation over the assay duration of $\sim 2$-weeks in ARMS cells with higher MET expression. Therefore, we examined the clonogenic potential of SPRY2 and MET silenced RMS cells employing adherence-dependent colony formation assay, where cells were cultured for a shorter, 1-week duration. SPRY2 and MET were required for clonal growth of RMS cells, and the number and size of colonies markedly reduced in RD (Fig. 5e- $\mathrm{e}^{\prime}$ and f) and SJRH30 (Fig. $5 \mathrm{k}-\mathrm{k}^{\prime}$ and $\mathrm{l}$ ) cells upon SPRY2 or MET knockdown. These findings indicate that SPRY2 and MET regulate metastatic and clonogenic potential in RMS cells.

Constitutive activation of MET inhibits differentiation while shRNA-mediated downregulation of MET induces differentiation in RMS $^{42}$. Therefore, we sought to determine whether depleting SPRY2, which reduces MET levels, could induce RMS cell differentiation. Consistent with earlier reports, MET knockdown induced RD (Fig. $6 \mathrm{c}-\mathrm{c}^{\prime}$ ) and SJRH30 (Fig. 6g-g') cells to change morphology, becoming elongated, multi-nucleate, and compared to controls myofiber-like (Fig. $6 \mathrm{a}-\mathrm{a}^{\prime}$ and $\mathrm{e}-\mathrm{e}^{\prime}$ ). Remarkably, SPRY2 depleted RD (Fig. 6b-b') and SJRH30 (Fig. $6 \mathrm{f}-\mathrm{f}^{\prime}$ ) cells formed similar differentiated structures (red arrows) by 144-h PsiRT. Thus, SPRY2 or MET depletion in RMS cells overcomes the differentiationblock, forming myofiber-like structures that are multinucleate, with linearly arranged nuclei characteristic of myofibers $^{43}$, and positive for MHC-EMBRYONIC, a myogenic differentiation marker (Fig. 6b", $c^{\prime \prime}, f^{\prime \prime}, g^{\prime \prime}$ ). MHC-EMBRYONIC expression was observed in control RD (Fig. 6a") and SJRH30 (Fig. 6e $\mathrm{e}^{\prime \prime}$ ) cells as reported previously ${ }^{11}$. However, compared to control cells, MHCEMBRYONIC was expressed at relatively higher levels in MET or SPRY2 depleted RMS cells 144-h PsiRT (Fig. 6d, h). Taken together, this indicates that silencing SPRY2, similar to MET downregulation, promotes differentiation in RMS cells.

\section{SPRY2 and MET promote ERK/MAPK signaling in RMS}

MAPK signaling regulates cell migration, motility and differentiation $^{44-50}$. Since MET is known to regulate both ERK/MAPK and p38/MAPK, we hypothesized that reduced migration and induction of differentiation upon MET or SPRY2 depletion in RMS could be mediated by these pathways ${ }^{20}$. Therefore, we assayed the activation of these two MAPK signaling branches in RMS, 144-h PsiRT, by western blotting.

We found that ERK/MAPK activation was significantly diminished in SPRY2 or MET depleted RD (Fig. 7a, b, upper panels) and SJRH30 (Fig. 7c, d, upper panels) cells. 


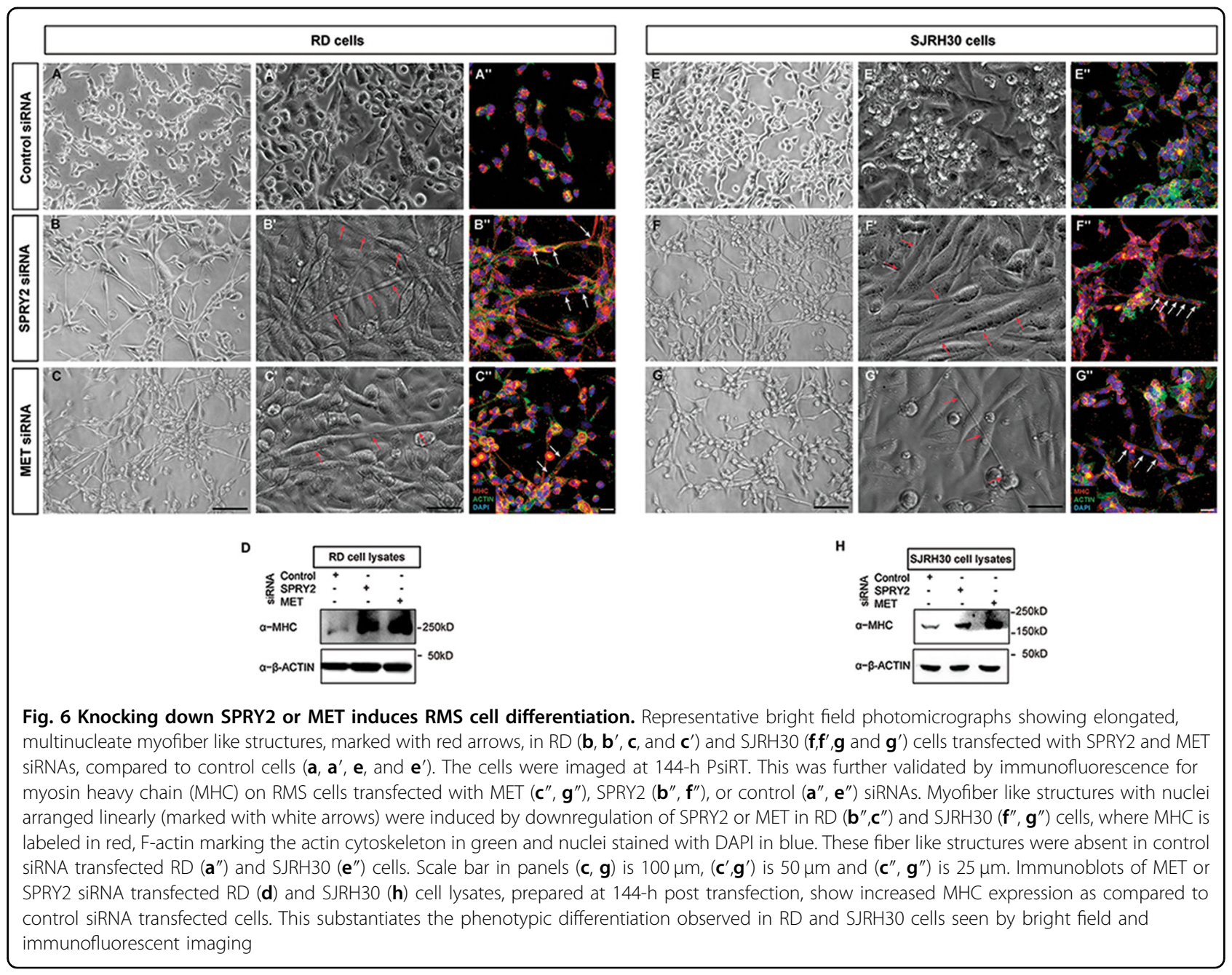

Interestingly, the inhibition of ERK/MAPK activation in RMS cells correlated with induction of differentiation observed at 144-h PsiRT. No significant difference in p38/ MAPK phosphorylation in MET or SPRY2 silenced RD cells was observed (Fig. 7a, b, lower panels). Thus, in RD cells, weakening of ERK/MAPK activation downstream of MET is sufficient to promote differentiation. In contrast, silencing MET or SPRY2 in SJRH30 cells significantly decreased p38/MAPK activation (Fig. 7c, d, lower panels). These findings indicate that although the phosphorylation status of p38/MAPK in ERMS and ARMS are different, highlighting the RMS sub-type specific requirement of p38 activity, the effect of MET and SPRY2 depletion is identical. This is similar to the requirement of p38 activation during early stages and its inhibition at late stages of myogenesis reported previously ${ }^{47,49}$.

To test whether reduced MAPK signaling resulting from silencing SPRY2 or MET is responsible for their impaired migratory potential, we assessed SJRH30 cell migration upon treatment with the MEK/ERK inhibitor U0126. Inhibition of MAPK signaling significantly impaired cell motility (Fig. 7e, f) and end point phosphorylated ERK levels showed that inhibition was efficiently maintained (Fig. 7g, h). Overall, SPRY2 engages with the MET receptor and stabilizes it, functioning to maintain downstream ERK signaling to sustain RMS migratory potential and prevent differentiation in ERMS and ARMS.

\section{Discussion}

MET is a RTK known to be dysregulated in RMS, demonstrated to be a promising therapeutic candidate $^{18,19,27,51}$. However, the reasons underlying misregulation of MET in RMS are incompletely understood. Using embryonal and alveolar RMS cell lines, we show for the first time that SPRY2 interacts with and stabilizes MET to sustain downstream signals that maintain the migratory and clonogenic potential and block differentiation in RMS.

We found that MET transcript and protein levels varied across RMS cell lines but were higher in ARMS compared to ERMS cells, which possibly correlates with its 


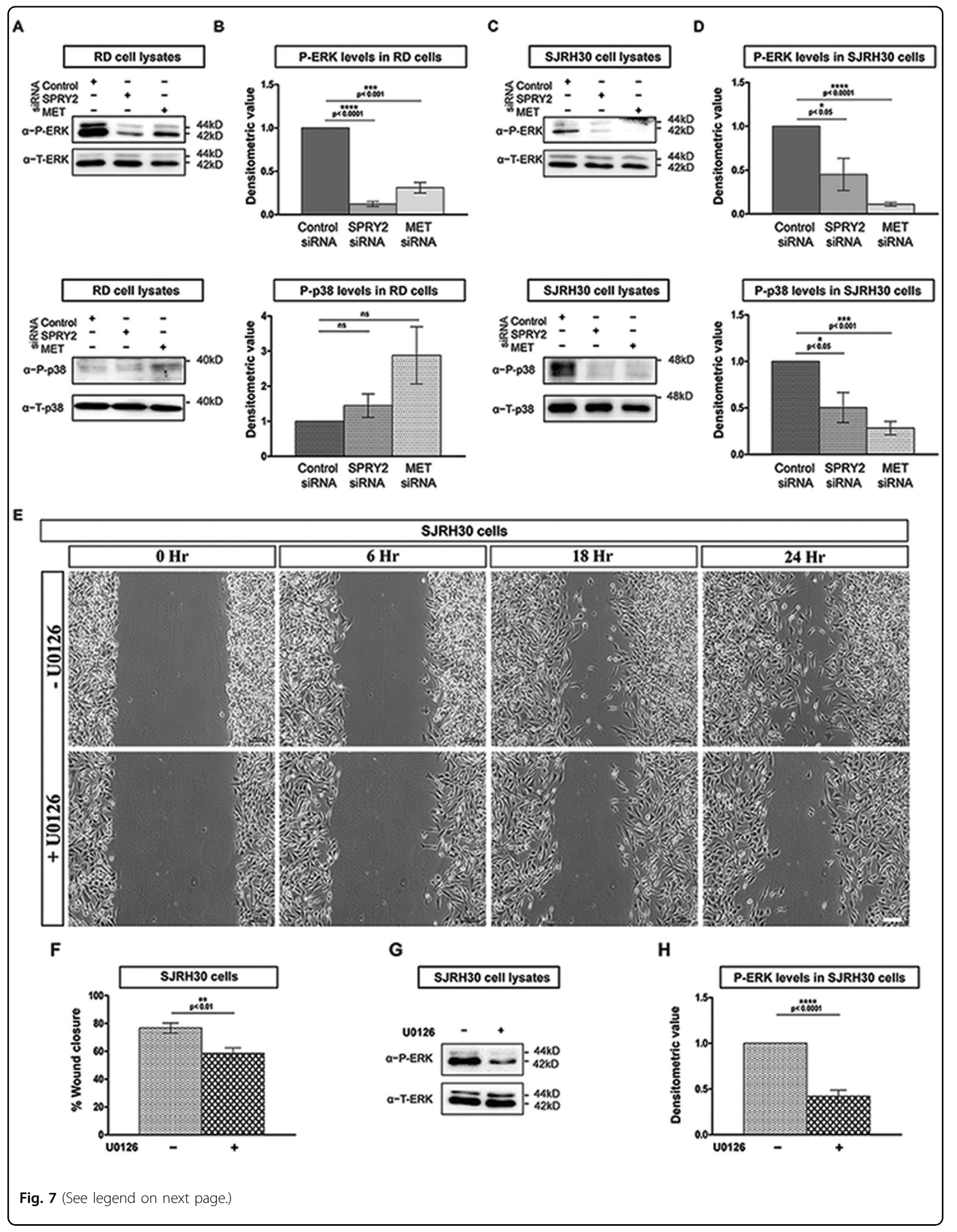




\begin{abstract}
(see figure on previous page)
Fig. 7 MET or SPRY2 knockdown causes similar alterations in MAP Kinase pathway activity in RMS cells. RMS cells were cultured for 144-h post transfection with MET, SPRY2, or control siRNA and lysates were separated by SDS-PAGE and analyzed by western blotting for MAP kinase pathway activation. Representative blots for levels of phosphorylated and total ERK1/2 (upper panels), as well as phosphorylated and total p38 (lower panels) detected using specific antibodies in RD (a) and SJRH30 (c) cells are shown. The ratios of pERK1/2 to T-ERK1/2 and p38 to total p38 were measured by densitometry (b, d). ERK activation is significantly reduced in both MET and SPRY2 silenced RD (b, upper panel) and SJRH30 (d, upper panel) cells when compared to control cells. However, phosphorylation of p38 is unchanged in RD cells (b, lower panel) but significantly reduced in SJRH30 cells (d, lower panel) between MET/SPRY2 and control siRNA transfections. Representative bright field micrographs of wound closure assays using SJRH30 cells, imaged at specific time points after incubation with (+) or without (-) the MEK/ERK inhibitor U0126 (e). Bar graphs show that wound closure in the monolayers of SJRH30 is significantly decreased in U0126 treated cells compared to untreated cells (f). Representative western blots for $\mathrm{p}$-ERK1/2 and total-ERK1/2 using cell lysates prepared at the end of the wound closure are shown (g). Densitometry shows that $\mathrm{p}$-ERK1/2 levels are significantly lower in U0126 treated SJRH30 cells when compared to untreated cells at the end of scratch assay (h). The graphical data are presented as mean \pm SEM of a minimum of three independent knockdown experiments. Scale bar in $\mathbf{e}$ is $100 \mu \mathrm{m}$
\end{abstract}

heightened aggressiveness and metastatic potential $^{19,26,38,42}$. Although HGF-MET signaling induced SPRY2 expression in leiomyosarcoma ${ }^{35}$, we found that SPRY2 levels were higher in ERMS cells that have low MET expression, compared to ARMS cells, suggesting additional mechanisms regulate SPRY2.

SPRY2 modulates MET signaling in cancer types such as leiomyosarcomas, hepatocarcinomas, and colonic adenocarcinomas $^{34-36}$. MET is overexpressed in non-small cell lung carcinoma (NSCLC), where miR-27a binds and downregulates MET and SPRY2 transcripts ${ }^{52}$. Intriguingly, downregulation of MET protein required concomitant overexpression of miR-27a and silencing of SPRY2, indicating that while miR-27a controls MET transcript levels, SPRY2 is critical in stabilizing MET protein in NSCLC ${ }^{52}$. SPRY2 is known to positively regulate MET levels in colon carcinoma, although it is unclear whether SPRY2 induces MET transcriptional activation or reduces MET degradation ${ }^{36}$. Thus, SPRY2 functions to maintain MET levels in multiple tumor types, although the precise mechanism is unclear. We observed that MET protein was significantly downregulated in SPRY2 depleted RMS cells, without altering MET transcript levels. This suggests that at least in RMS, SPRY2 does not regulate MET expression transcriptionally, but presence of SPRY2 inhibits MET protein degradation resulting in its stabilization. SPRY2 knockdown resulted in MET degradation preferentially via the proteasomal pathway in ERMS and lysosome-mediated pathway in ARMS, indicating that multiple degradatory mechanisms regulate MET, as reported previously ${ }^{39}$. Since we found that MET and SPRY2 interact with each other, it is likely that this interaction results in MET stabilization. Interestingly, this interaction also stabilizes SPRY2, since MET depletion led to a reduction in SPRY2 protein levels without affecting transcript levels in ARMS. One possibility is that the MET-SPRY2 interaction renders motifs on both proteins that target them for degradation, such as ubiquitination, inaccessible.
Although there are differences between embryonal and alveolar RMS, both tumor sub-types share similar features such as tumor cells exhibiting muscle cell characteristics and inhibition of differentiation ${ }^{9-12}$. Previous studies indicate that inhibition of MET reduces the metastatic potential and overrides the differentiation block in RMS cells $^{26,27}$. Our finding that SPRY2 stabilizes MET, and depletion of SPRY2 leads to MET downregulation indicates that loss of SPRY2 should lead to decreased metastatic potential and permit differentiation of RMS cells. We found this to be the case where SPRY2 depletion led to decreased migration, and induction of differentiation. Also, SPRY2 and MET conferred clonogenic potential on RMS cells and silencing either significantly reduced colony forming ability of these cells. Whether SPRY2 plays MET independent roles in regulating RMS migration, differentiation or clonogenic potential is unknown, and could be investigated by overexpressing SPRY2 in MET depleted RMS cells.

We did not observe any effect of MET silencing on cellular proliferation in RMS cells. A previous study reported that RMS proliferation rate decreased upon downregulation of $\mathrm{MET}^{27}$. We observed a significant increase in cell death in ERMS cells upon MET knockdown, but not in ARMS, compared to a study which reported increased cell death in both ERMS and ARMS ${ }^{27}$. This could be due to differences in experimental set up, where we analyzed cells six days PsiRT, whereas the previous study used a doxycycline-inducible conditional lentiviral shRNA construct targeting MET over five days to study proliferation and cell death ${ }^{27}$. We found that downregulation of SPRY2 caused significantly reduced proliferation in ERMS and significantly decreased cell death in ARMS, indicating that SPRY2 might have MET independent functions in RMS. The varying trends of cell death and proliferation seen in MET or SPRY2 silenced RD and SJRH30 cells in this study reflect the intrinsic molecular differences between the two RMS sub-types. 

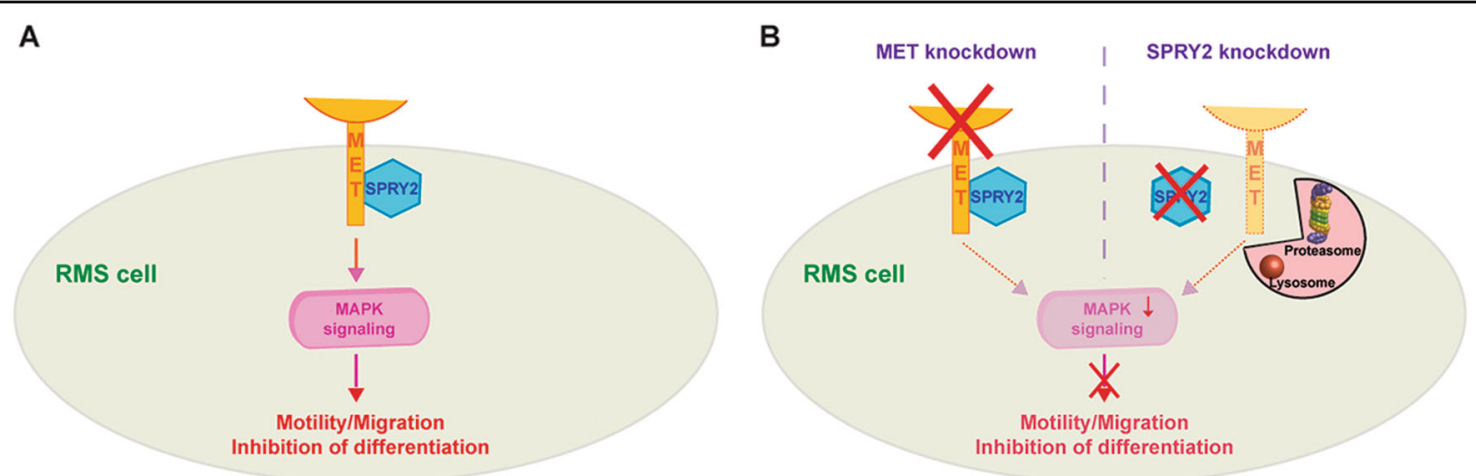

Fig. 8 SPRY2 is required to stabilize and regulate signaling downstream of the MET receptor. MAPK signaling transduced by aberrant MET activation and sustained by SPRY2 mediated stabilization of the MET receptor inhibits myogenic differentiation and confers metastatic characteristics on RMS cells (a). Silencing MET induces differentiation and reduces the metastatic potential of RMS cells by dampening the MAPK signaling (b). Silencing SPRY2 decreases MET stability and results in reduced MAPK signaling, thereby recapitulating the effects of MET silencing in RMS cells (b). Proteasomal degradation is the preferred pathway of MET degradation in ERMS (RD) cells while lysosome mediated degradation is the preferred pathway of MET degradation in ARMS (SJRH30) cells. MET degradation by these pathways is prevented when SPRY2 is bound to MET

Two pathways reported to function downstream of MET are the ERK (p44/42)/MAPK and p38/MAPK pathways $^{20}$. While the ERK (p44/42)/MAPK pathway regulates cell migration downstream of MET receptor, both MAPK pathways are essential for myogenic differentiation $^{44-49}$. Inhibition of ERK/MAPK signal is required for the myogenic program to proceed normally, whereas p38 activation is required at early stages but needs to be suppressed at later stages for myogenic differentiation $^{46,47,49}$. Our results suggest that ERK/MAPK pathway is the direct target of MET and SPRY2 in RMS, and the decreased metastatic potential and induction of differentiation observed upon MET or SPRY2 depletion is mediated by this pathway. Interestingly, we also observed a significant decrease in p38/MAPK activation in ARMS but not ERMS cells upon MET or SPRY2 depletion, which might be because ARMS tumor cells resemble the developing fetal muscles compared to ERMS which exhibit characteristics of embryonic muscles ${ }^{11}$. At later stages of myogenesis, p38/MAPK is suppressed, which may not be taking place in ARMS cells, contributing to the lack of differentiation ${ }^{49}$. ERMS cells migrated at a slower rate (42-h) compared to ARMS cells (24-h) in wound-healing assays, agreeing with reports that ARMS is relatively more metastatic and aggressive compared to ERMS $^{26,38}$.

We find that in addition to MET, SPRY2, a bimodal regulator of RTK signaling also exhibits elevated levels of expression in RMS. However, SPRY2 has never been shown to directly interact with the RTK itself to regulate it or downstream signaling. Here, we show that SPRY2 colocalizes and interacts with MET, stabilizing and preventing MET degradation, which leads to elevated ERK/ MAPK signaling (Fig. 8). All of this facilitates the maintenance of increased migratory potential and inhibition of differentiation, characteristic of RMS (Fig. 8). The identification of this unique molecular interaction between SPRY2 and MET that functions to regulate the oncogenic properties of RMS raises the possibility of leveraging it for future therapeutic interventions in RMS and other tumor types where MET is aberrantly activated.

\section{Materials and Methods \\ Cell culture}

Human RMS cells of embryonal (RD; Cat\# CCL-136) and alveolar (SJRH30; Cat\# CRL-2061) sub-type were purchased from American Type Cell Culture (ATCC) (Manassas, VA, USA). ARMS cell lines - RH4, RH28 and RH41 were kindly provided by Dr. Peter J. Houghton (Nationwide Children's Hospital, Columbus, Ohio, USA). These cell lines were grown and maintained as recommended, in Dulbecco Modified Eagle Medium (DMEM) (Gibco, Thermo Fisher Scientific, Waltham, MA, USA; Cat\# 11995065) supplemented with 10\% (v/v) fetal bovine serum (Sigma, St. Louis, Missouri, USA; Cat\# F2442) and $1 \%$ penicillin-streptomycin (i.e., $100 \mathrm{U} / \mathrm{ml}$, Gibco; Cat\# $15140122)$ at $37{ }^{\circ} \mathrm{C}, 5 \% \mathrm{CO}_{2}$ and $95 \%$ humidity.

For expression analyses at transcript and protein levels, $\sim 60,000$ and 3,00,000 cells were plated in each well of 24well (Nunc, Roskilde, Denmark; Cat\# 142485) and 6-well plates (Corning, New York, USA; Cat\# 3506), respectively and allowed to grow to $\sim 80 \%$ confluence. Cells from 4 wells of a 24-well plate and one 6-well plate were harvested per replicate, to isolate RNA and prepare protein lysates, respectively. These experiments were carried out in triplicates. For knockdown experiments, reverse transfection was performed in RD and SJRH30 cells. Briefly, 60,000 cells were seeded in each well of a 24-well plate that was pre-layered with transfection mix comprising $100 \mu \mathrm{l}$ Opti-MEM (Gibco; Cat\# 31985070), $10 \mathrm{nM}$ 
of MET or SPRY2 or control siRNAS (Silencer Select siRNAs, Ambion, Thermo Fisher Scientific; Cat\# s8702, s20028 and 4390847, respectively) and $2 \mu \mathrm{l}$ Lipofectamine RNAiMAX (Invitrogen, Thermo Fisher Scientific; Cat\# 13778150). At 48 and 144-h post siRNA transfection (PsiRT), 4 wells of a 24-well plate and one 24-well plate were harvested per replicate to isolate RNA and prepare protein extracts, respectively. ON-TARGETplus SMARTpool siRNAs targeting human MET (10 nM) and SPRY2 (50 nM) (Dharmacon, Cambridge, UK; Cat\# L-003156-00-0005 and L-005206-00-0005, respectively) were used to validate the knockdown observed using the Silencer Select siRNAs (Ambion). These experiments were performed a minimum of three times. All experiments requiring knockdown were subsequently performed using Ambion Silencer Select siRNAs. Where specified, RMS cells were incubated in the presence of lysosomal inhibitor Bafilomycin A1 (100 nM for 4-h, Sigma; Cat\# B1793), proteasomal inhibitor MG132 (25 $\mu \mathrm{M}$ for 6-h; Cayman Chemical Company, Michigan, Ann Arbor, USA; Cat\#1001268), or vehicle control, 96-h PsiRT. For immunofluorescence studies, RMS cells were seeded onto gelatin-coated coverslips (Neuvitro, Vancouver, WA, USA; Cat\# GG-12) in 24-well plates (Nunc; Cat\# 142485). Approximately 30,000 cells/well were plated for immunofluorescence based expression and colocalization experiments, whereas $\sim 50,000$ cells/well were reverse transfected and seeded, as described above, for analysis of knockdown induced differentiation of RMS cells.

\section{RNA isolation, CDNA synthesis, and qPCR}

RNA was isolated from RMS cells harvested as described in the cell culture section above, using RNeasy mini kit (Qiagen, Hilden, Germany; Cat\# 74106). cDNA was synthesized from $1 \mu \mathrm{g}$ of RNA using SuperScript III reverse transcriptase (Invitrogen, Cat\# 18080-044) and oligo (dT) (Invitrogen, Cat\# 58862). MET and SPRY2 transcripts were quantified by quantitative real time polymerase chain reaction (qPCR) using SYBR Green with ROX as internal reference (Applied Biosystems, Thermo Fisher Scientific; Cat\# 4367659) using the ABI 7500 Fast Real Time PCR system (Applied Biosystems). The primers used for gene expression profiling in different RMS cell lines are listed in Supplementary Table 1. GAPDH and HPRT were used as endogenous controls or reference genes for normalizing expression of target genes (that is $M E T$ and $S P R Y 2)^{53,54}$. RNA from mesenchymal stem cells, closest appropriate control for RMS cells that are thought to originate from cells of the mesenchymal ori$\operatorname{gin}^{14}$, was used as a control or calibrator. All the qPCR assays were performed in a final reaction volume of $20 \mu \mathrm{l}$ using $100 \mathrm{nM}$ forward and reverse primers of the respective genes, as recommended by ABI. Each reaction was performed in triplicates. The expression of target genes in the siRNA transfected RMS cells was normalized to the levels in control siRNA transfected cells ${ }^{55}$. Three replicate cDNA samples were used for quantifying endogenous expression of target genes in different cell lines. Three matched sets of MET or SPRY2 and control siRNA transfected samples were used as biological replicates for performing expression analysis and the error bar represents \pm standard error of the mean (SEM).

\section{Co-immunoprecipitation}

RMS cells were lysed in ice-cold lysis buffer $(200 \mathrm{mM}$ Tris, pH7.4; $120 \mathrm{mM} \mathrm{NaCl} ; 0.2 \%$ sodium deoxycholate; $0.1 \%$ SDS; 1 mM EGTA, 1\% Triton-X 100) supplemented with $1 \times$ protease inhibitor (Sigma; Cat\# P8340). Debris was removed by centrifugation at $6000 \mathrm{rpm}$ for $5 \mathrm{~min}$ at 4 ${ }^{\circ} \mathrm{C}$ and the lysates obtained were quantified using BCA Protein Assay kit (Pierce, Thermo Fisher Scientific; Cat\# 23225) as per manufacturer's protocol. Approximately $500 \mu \mathrm{g}$ protein was incubated with Protein A Sepharose 4 Fast flow beads (GE Healthcare Life Sciences, Chicago, IL, USA; Cat\# 17-5280-01) for 1 -h at $4{ }^{\circ} \mathrm{C}$ on a tube mixer, followed by centrifugation to remove the beads and the proteins that were bound non-specifically. Subsequently, the pre-cleared lysates were used to immunoprecipitate MET-conjugated proteins by incubation with MET antibody overnight at $4{ }^{\circ} \mathrm{C}$ on a tube mixer. IgG raised in rabbit was used as a control antibody. The antibodybound proteins were then incubated with Protein A Sepharose 4 Fast flow beads for 6 -h at $4{ }^{\circ} \mathrm{C}$ on a tube mixer to capture the immunocomplexes. Immunoprecipitates were washed thrice using ice-cold lysis buffer and eluted by boiling in $2 \times$ Laemmli sample buffer at $95^{\circ} \mathrm{C}$ for $3 \mathrm{~min}$. The immunoprecipitates were subjected to western analysis as described below. Antibodies and the concentrations used for immunoprecipitation are listed in Supplementary Table 2.

\section{Immunoblotting}

Protein lysates were prepared from RMS cells that were plated, cultured, and harvested at required time points as explained above in the cell culture section. Briefly, the cells were harvested by aspirating the medium from the wells, trypsinizing and centrifuging at $6000 \mathrm{rpm} / 4{ }^{\circ} \mathrm{C} / 6$ $\min$. Subsequently, cells were washed with ice-cold $1 \times$ DPBS (Gibco; Cat\# 14190-144) twice, followed by centrifugation. Cells were lysed in ice-cold radioimmunoprecipitation assay (RIPA) buffer (Sigma; Cat\# R0278-50) containing protease inhibitor (Sigma; Cat\# P8340) and phosphatase inhibitor (Cell Signaling Technology, Danvers, MA, USA; Cat\# 58705), each at 1:100 dilution. The protein samples were quantified using BCA Protein Assay kit (Thermo Fisher Scientific; Cat\# 23225) as per manufacturer's protocol. Equal concentrations of 
protein extracts were separated by $10 \%$ or $12 \%$ SDSPAGE, depending on the molecular weight of the protein to be detected, followed by transfer to PVDF membrane (Millipore; Billerica, MA, USA; Cat\# iPVH00010) at $4{ }^{\circ} \mathrm{C}$ overnight. Subsequently, the membranes were blocked in $5 \%$ skimmed milk for 5 -h, incubated overnight in primary antibody at $4{ }^{\circ} \mathrm{C}$, and 2-h in appropriate HRP-conjugated secondary antibody at room temperature. Signals were detected using the Luminata Forte HRP substrate (Millipore; Cat\# WBLUF0100). Blots were imaged using ImageQuant LAS 4000 and densitometric analyses were performed using and ImageQuant TL v7.0 software (GE Healthcare Life Sciences). For densitometric analyses, reference genes used as loading controls for normalization were $\beta$-Actin, GAPDH, or $\beta$-Tubulin. Data from a minimum of three independent replicates were plotted as an average, with the error bars representing \pm SEM. Antibodies and the concentrations used for western blotting are listed in Supplementary Table 2.

\section{Wound closure and transwell migration assays}

RMS cells were seeded in 6-well plates and reverse transfected with MET, SPRY2, or control siRNAs respectively, as described above in the cell culture section. A seeding cell density of $\sim 2.5 \times 10^{5}$ cells/well was used so that the cells attain about $90 \%$ confluence by around $96-\mathrm{h}$ post siRNA transfection. Subsequently, the media containing the transfection mix was aspirated out of each well, preserved in labeled tubes and the cell monolayers were scratched carefully with a sterile $200 \mu$ pipette tip. The wells were washed gently with $1 \times$ DPBS (Gibco; Cat\# 14190-144) thrice to get rid of the scraped floating cells and the preserved transfection mix was added back to the respective wells. The scratch was imaged immediately for the zero-time point (0) using an inverted microscope (Nikon Eclipse TS100, Minato, Tokyo, Japan). Subsequently, the cells were incubated at $37^{\circ} \mathrm{C}, 5 \% \mathrm{CO}_{2}$ and 95\% humidity, and the scratch monitored and documented over time at the same positions. A mark was made on the plastic plate as a reference point to ensure that the same area was imaged every time over the time course of the experiment ${ }^{41}$. Imaging was performed until the time point $(t)$ when the wound closed in the control siRNA transfected RMS cells. The images were used to measure the scratched area at zero-time point $\left(\mathrm{A}_{0}\right)$ and at the time point of closure $\left(A_{t}\right)$, using a macro (freely available at http://rsb.info.nih.gov/ij/) written at Montpellier RIO Imaging $^{56}$ for ImageJ software ${ }^{57,58}$. Percentage wound closure was calculated using the formula $\left(\left(\mathrm{A}_{0}-\mathrm{A}_{t}\right) /\right.$ $\left.A_{0}\right) \times 100$. Wound closure assays were also performed on SJRH30 cells in the presence and absence of MEK/ERK inhibitor U0126 (Cell Signaling Technology; Cat\# 9903). The wound healing assays were performed independently a minimum of five times for each transfection.
Transwell migration assay was performed to evaluate the effect of MET and SPRY2 knock-down on metastatic potential of RMS cells. Briefly, 96-h post transfection with MET, SPRY2 or control siRNA, RMS cells were trypsinized and re-suspended in serum free medium. Approximately 80,000 RD and 60,000 SJRH30 cells were seeded in transwell inserts $(8 \mu \mathrm{m}$ pore size, Corning; Cat\#3464), and placed into 24-well cell culture plates containing DMEM with $10 \% \mathrm{FBS}$. After incubation at $37^{\circ}$ $\mathrm{C}$ for 24-h, non-migrating cells on the upper surface of the membrane were removed with cotton swabs and migrated cells at the base of the inserts were fixed in $4 \%$ PFA ( $5 \mathrm{~min})$, permeabilized with methanol $(20 \mathrm{~min})$ and stained with $2 \%$ crystal violet dye $(15 \mathrm{~min})$. Cells were counted manually and imaged using a stereomicroscope (Leica S8 APO, Houston, Texas, USA). Percentage cell migration was calculated relative to control siRNA transfected RMS cells. The transwell migration assays were performed independently a minimum of three times for each transfection. Graphical data for the wound closure and transwell migration assays are represented as mean \pm SEM.

\section{Clonogenic assays}

Anchorage-independent colony forming ability of MET or SPRY2 silenced RMS cells was examined by soft agar assay. A base layer was prepared by pipetting $2 \mathrm{ml} 0.7 \%$ agar (BD Bacto agar, Fisher Scientific; Cat\# 214010) in 10\%FBS/1\%Pen-Strep/DMEM, in 6-well plates. This base layer was overlaid with $0.35 \%$ agar with 6000 siRNA transfected RMS cells (96-h PsiRT) suspended in it. The cells were then cultured for about 2-weeks. $1 \mathrm{ml}$ culture medium was supplemented to each well every 5 days to prevent desiccation of agar. Colonies were fixed and stained using $0.05 \%$ crystal violet in neutral buffered formalin for 1-h. Colonies were counted manually and imaged using a stereomicroscope (Leica S8 APO).

For anchorage-dependent colony formation assay MET, SPRY2, or control siRNA transfected RMS cells were plated 96-h PsiRT in 6 well plates at 3000 cells/well and cultured for 8 days. Colonies were washed twice with $1 \times \mathrm{PBS}$, fixed in ice cold methanol $(20 \mathrm{~min})$ and stained with $2 \%$ crystal violet $(\sim 30 \mathrm{~min})$. The wells were imaged using a digital camera and representative individual colonies form different transfections were imaged using a stereomicroscope (Leica S8 APO). Colony forming units or colonies comprising $>30$ cells were counted manually under the stereomicroscope.

Both the clonogenic assays were performed independently a minimum of three times for each transfection and graphical data is represented as mean \pm SEM. 


\section{Immunofluorescence and microscopy}

RMS cells for immunofluorescence were grown as detailed in the cell culture section above. The cells were fixed in $4 \%$ paraformaldehyde (PFA) for $20 \mathrm{~min}$ and washed with $\mathrm{PBS}$ following which they were blocked with $5 \%$ goat serum (BioAbChem, Ladson, SC, USA; Cat\# 720480) in PBS containing $0.1 \%$ Triton-X-100 for 1 -h at room temperature (RT). The cells were incubated overnight at $4{ }^{\circ} \mathrm{C}$ with appropriate concentrations of respective primary antibodies listed in Supplementary Table 2, washed thrice with PBS, incubated with the appropriate secondary antibody for 2-h at RT, and washed with PBS. In experiments where signal amplification was required for detection of the target protein, cells were incubated with a biotin conjugated secondary antibody for 2-h at RT and then with fluorophore conjugated to streptavidin for 1 -h at RT, washed thrice with PBS, rinsed in distilled water and allowed to dry. Coverslips were mounted with DAPI Fluoromount-G (Southern Biotech, Birmingham, AL, USA; Cat\# 0100-20). Images were captured using the Leica TCS SP5 or SP8 confocal microscopes using identical settings for a particular staining across treatments for all replicates.

The colocalization between SPRY2 and MET was quantified using the colocalization tool in the Leica Application Suite X (LAS X) software, with the threshold and background parameters for both channels set to $60 \%$ and $20 \%$, respectively. Analysis was performed on 17 individual cells, in three different images, showing SPRY2MET colocalization and the mean Pearson's correlation coefficient was calculated from these regions of interest $(\mathrm{ROIs})^{40}$.

\section{Statistical analyses}

Experimental data was plotted in GraphPad Prism 5 (GraphPad Software Inc., CA, USA) and analyzed using the parametric, paired t-test using the SigmaPlot12.5 (Systat Software Inc., Germany). All graphical data is represented as mean \pm SEM, and each experiment was performed at least in triplicates. The $p$-value is indicated on the graph along with asterisks and $p$-value $\leq 0.05$ is considered significant. The level of significance is indicated as ${ }^{*} p<0.05 ;{ }^{* * *} p<0.01 ;{ }^{* * * *} p<0.001 ;{ }^{* * * * * *} p<0.0001$.

\footnotetext{
Acknowledgements

This work was supported by funding from the Department of Biotechnology, Government of India, through a Pilot Project in Cancer Biology grant (sanction number 6242-P63/RGCB/PMD/DBT/SMJM/2015), and a Wellcome Trust-DBT India Alliance intermediate fellowship (IA///13/1/500872) to S.J.M. We also acknowledge core funds provided by the Regional Centre for Biotechnology (RCB). M.S. is funded by a Young Investigator award from RCB. We thank Prof. Peter J. Houghton at the Greehey Children's Cancer Research Institute, University of Texas Health Science Center, San Antonio, USA, for generously providing us some of the alveolar rhabdomyosarcoma cell lines used in this study. We thank Dr. Prasenjit Guchhait for providing some antibodies used in this work. We also acknowledge the imaging and central instrumentation
}

facilities at RCB. We acknowledge the support of DBT e-Library Consortium (DeLCON) for providing access to e-resources.

\section{Conflict of interest}

The authors declare that they have no conflict of interest.

\section{Publisher's note}

Springer Nature remains neutral with regard to jurisdictional claims in published maps and institutional affiliations.

Supplementary Information accompanies this paper at (https://doi.org/ 10.1038/s41419-018-0261-2).

Received: 29 July 2017 Revised: 27 November 2017 Accepted: 14 December 2017

Published online: 14 February 2018

\section{References}

1. American Cancer Society. Cancer Facts \& Figures. https://www.cancer.org/ research/cancer-facts-statistics/all-cancer-facts-figures/cancer-facts-figures2017.html (2017).

2. Hawkins, D. S., Spunt, S. L. \& Skapek, S. X. Children's Oncology Group's 2013 blueprint for research: Soft tissue sarcomas. Pediatr. Blood. Cancer 60, 1001-1008 (2013).

3. American Cancer Society. Incidence rates. https:/www.cancer.org/cancer/ rhabdomyosarcoma/about/key-statistics.html (2014).

4. Ognjanovic, S., Linabery, A. M., Charbonneau, B. \& Ross, J. A. Trends in childhood rhabdomyosarcoma incidence and survival in the United States, 1975-2005. Cancer 115, 4218-4226 (2009).

5. Fletcher, C. D., Organization WH. WHO classification of tumours of soft tissue and bone:[this book reflects the views of a working group that convened for a consensus and editorial meeting at the University of Zurich, Switzerland, 18-20 April 2012]. Agency for Research on Cancer (2013).

6. Perez, E. A. et al. Rhabdomyosarcoma in children: a SEER population based study. J. Surg. Res. 170, e243-e251 (2011).

7. Parham, D. M. \& Ellison, D. A. Rhabdomyosarcomas in adults and children: an update. Arch. Pathol. Lab. Med. 130, 1454-1465 (2006).

8. Sokolowski, E., Turina, C., Kikuchi, K., Langenau, D. \& Keller, C. Proof-of-concept rare cancers in drug development: the case for rhabdomyosarcoma. Oncogene 33, 1877-1889 (2014)

9. Merlino, G., Helman, L. J. Rhabdomyosarcoma-working out the pathways. Oncogene. 18, 5340-5348 (1999).

10. Sebire, N. \& Malone, M. Myogenin and MyoD1 expression in paediatric rhabdomyosarcomas. J. Clin. Pathol. 56, 412-416 (2003).

11. Tonin, P. N., Scrable, H., Shimada, H. \& Cavenee, W. K. Muscle-specific gene expression in rhabdomyosarcomas and stages of human fetal skeletal muscle development. Cancer Res. 51, 5100-5106 (1991).

12. Morotti, R. A. et al. An immunohistochemical algorithm to facilitate diagnosis and subtyping of rhabdomyosarcoma: the Children's Oncology Group experience. Am. J. Surg. Pathol. 30, 962-968 (2006).

13. Keller, $\mathrm{C}$. et al. Alveolar rhabdomyosarcomas in conditional Pax3: Fkhr mice: cooperativity of Ink4a/ARF and Trp53 loss of function. Genes Dev. 18, 2614-2626 (2004).

14. Charytonowicz, E., Cordon-Cardo, C., Matushansky, I. \& Ziman, M. Alveolar rhabdomyosarcoma: is the cell of origin a mesenchymal stem cell? Cancer Lett. 279, 126-136 (2009).

15. Tiffin, N., Williams, R., Shipley, J. \& Pritchard-Jones, K. PAX7 expression in embryonal rhabdomyosarcoma suggests an origin in muscle satellite cells. $\mathrm{Br}$. J. Cancer 89, 327-332 (2003).

16. Bladt, F., Riethmacher, D., Isenmann, S., Aguzzi, A. \& Birchmeier, C. Essential role for the c-met receptor in the migration of myogenic precursor cells into the limb bud. Nature 376, 768-771 (1995).

17. Dietrich, S. et al. The role of SF/HGF and c-Met in the development of skeletal muscle. Development 126, 1621-1629 (1999).

18. Ferracini, R. et al. Retrogenic expression of the MET proto-oncogene correlates with the invasive phenotype of human rhabdomyosarcomas. Oncogene $\mathbf{1 2}$, 1697-1705 (1996). 
19. Epstein, J. A., Shapiro, D. N., Cheng, J., Lam, P. \& Maas, R. L. Pax3 modulates expression of the c-Met receptor during limb muscle development. Proc. Natl Acad. Sci. USA 93, 4213-4218 (1996).

20. Trusolino, L., Bertotti, A. \& Comoglio, P. M. MET signalling: principles and functions in development, organ regeneration and cancer. Nat. Rev. Mol. Cell. Biol. 11, 834-848 (2010).

21. Cooper, C. S. et al. Molecular cloning of a new transforming gene from a chemically transformed human cell line. Nature 311, 29-33 (1984).

22. Cornelison, D. \& Wold, B. J. Single-cell analysis of regulatory gene expression in quiescent and activated mouse skeletal muscle satellite cells. Dev. Biol. 191, 270-283 (1997)

23. Tatsumi, R., Anderson, J. E., Nevoret, C. J., Halevy, O. \& Allen, R. E. HGF/SF is present in normal adult skeletal muscle and is capable of activating satellite cells. Dev. Biol. 194, 114-128 (1998).

24. Webster, M. T. \& Fan, C.-M. C-MET regulates myoblast motility and myocyte fusion during adult skeletal muscle regeneration. PLOS ONE 8, e81757 (2013).

25. Cen, L. et al. Phosphorylation profiles of protein kinases in alveolar and embryonal rhabdomyosarcoma. Mod. Pathol. 20, 936-946 (2007).

26. Miekus, $\mathrm{K}$. et al. The decreased metastatic potential of rhabdomyosarcoma cells obtained through MET receptor downregulation and the induction of differentiation. Cell Death Dis. 4, e459 (2013).

27. Taulli, R. et al. Validation of met as a therapeutic target in alveolar and embryonal rhabdomyosarcoma. Cancer Res. 66, 4742-4749 (2006).

28. Ledda, F. \& Paratcha, G. Negative regulation of receptor tyrosine kinase (RTK) signaling: a developing field. Biomark. Insights 2, 45 (2007).

29. Edwin, F., Anderson, K., Ying, C. \& Patel, T. B. Intermolecular interactions of Sprouty proteins and their implications in development and disease. Mol. Pharmacol. 76, 679-691 (2009).

30. Masoumi-Moghaddam, S., Amini, A. \& Morris, D. L. The developing story of Sprouty and cancer. Cancer Metastas-. Rev. 33, 695-720 (2014).

31. Egan, J. E., Hall, A. B., Yatsula, B. A. \& Bar-Sagi, D. The bimodal regulation of epidermal growth factor signaling by human Sprouty proteins. Proc. Natl Acad. Sci. USA 99, 6041-6046 (2002).

32. Wong, E. S. M. et al. Sprouty2 attenuates epidermal growth factor receptor ubiquitylation and endocytosis, and consequently enhances Ras/ERK signalling. EMBO J. 21, 4796-4808 (2002)

33. Yusoff, P. et al. Sprouty2 inhibits the Ras/MAP kinase pathway by inhibiting the activation of Raf. J. Biol. Chem. 277, 3195-3201 (2002).

34. Lee, S. A. et al. Synergistic role of sprouty2 inactivation and c-Met up-regulation in mouse and human hepatocarcinogenesis. Hepatology 52, 506-517 (2010).

35. Lee, C.-C. et al. Overexpression of sprouty 2 inhibits HGF/SF-mediated cell growth, invasion, migration, and cytokinesis. Oncogene 23, 5193-5202 (2004).

36. Holgren, C. et al. Sprouty-2 controls c-Met expression and metastatic potential of colon cancer cells: sprouty/c-Met upregulation in human colonic adenocarcinomas. Oncogene 29, 5241-5253 (2010).

37. Ozaki, K. et al. ERK pathway positively regulates the expression of Sprouty genes. Biochem. Biophys. Res. Commun. 285, 1084-1088 (2001).

38. Diomedi-Camassei, F. et al. Clinical significance of CXC chemokine receptor-4 and c-Met in childhood rhabdomyosarcoma. Clin. Cancer Res. 14, 4119-4127 (2008).

39. Hammond, D. E., Urbe, S., Vande Woude, G. F. \& Clague, M. J. Down-regulation of MET, the receptor for hepatocyte growth factor. Oncogene 20, 2761-2770 (2001).
40. Dunn, K. W., Kamocka, M. M. \& McDonald, J. H. A practical guide to evaluating colocalization in biological microscopy. Am. J. Physiol.-Cell Physiol. 300 C723-C742 (2011)

41. Liang, C.-C., Park, A. Y. \& Guan, J.-L. In vitro scratch assay: a convenient and inexpensive method for analysis of cell migration in vitro. Nat. Protoc. 2, 329-333 (2007).

42. Skrzypek, K. et al. Constitutive activation of MET signaling impairs myogenic differentiation of rhabdomyosarcoma and promotes its development and progression. Oncotarget 6, 31378-31398 (2015).

43. Yoshida, N., Yoshida, S., Koishi, K., Masuda, K. \& Nabeshima, Y-i. Cell heterogeneity upon myogenic differentiation: down-regulation of MyoD and Myf-5 generates 'reserve cells'. J. Cell. Sci. 111, 769-779 (1998)

44. Birchmeier, C., Birchmeier, W., Gherardi, E. \& Woude, G. F. V. Met, metastasis, motility and more. Nat. Rev. Mol. Cell. Biol. 4, 915-925 (2003).

45. Potempa, S. \& Ridley, A. J. Activation of both MAP kinase and phosphatidy linositide 3-kinase by Ras is required for hepatocyte growth factor/scatter factor-induced adherens junction disassembly. Mol. Biol. Cell 9, 2185-2200 (1998).

46. Bennett, A. M. \& Tonks, N. K. Regulation of distinct stages of skeletal muscle differentiation by mitogen-activated protein kinases. Science 278, 1288-1291 (1997).

47. Puri, P. L. et al. Induction of terminal differentiation by constitutive activation of p38 MAP kinase in human rhabdomyosarcoma cells. Genes Dev. 14, 574-584 (2000).

48. Rossi, S., Stoppani, E., Puri, P. L. \& Fanzani, A. Differentiation of human rhabdomyosarcoma RD cells is regulated by reciprocal, functional interactions between myostatin, p38 and extracellular regulated kinase signalling pathways. Eur. J. Cancer 47, 1095-1105 (2011).

49. Weston, A. D. Sampaio, A. V., Ridgeway, A. G. \& Underhill, T. M. Inhibition of p38 MAPK signaling promotes late stages of myogenesis. J. Cell Sci. 116, 2885-2893 (2003).

50. $\mathrm{Wu}, \mathrm{Z}$. et al. p38 and extracellular signal-regulated kinases regulate the myogenic program at multiple steps. Mol. Cell Biol. 20, 3951-3964 (2000).

51. Hou, J. et al. Inhibition of phosphorylated c-Met in rhabdomyosarcoma cell lines by a small molecule inhibitor SU11274. J. Transl. Med. 9, 64 (2011).

52. Acunzo, M. et al. Cross-talk between MET and EGFR in non-small cell lung cancer involves miR-27a and Sprouty2. Proc. Natl Acad. Sci. USA 110 8573-8578 (2013).

53. Saini, M., Jha, A. N., Abrari, A. \& Ali, S. Expression of proto-oncogene KIT is upregulated in subset of human meningiomas. Bmc. Cancer 12, 212 (2012).

54. Zhang, M., Truscott, J. \& Davie, J. Loss of MEF2D expression inhibits differentiation and contributes to oncogenesis in rhabdomyosarcoma cells. Mol. Cancer 12, 150 (2013).

55. Livak, K. J. \& Schmittgen, T. D. Analysis of relative gene expression data using real-time quantitative $P C R$ and the $2-\triangle \triangle C T$ method. Methods 25, 402-408 (2001).

56. Baecker, V. ImageJ macro tool sets for biological image analysis. In ImageJ User and Developer Conference 2012. Luxembourg: Centre de Recherche Public Henri Tudor (2012).

57. Schneider, C. A., Rasband, W. S. \& Eliceiri, K. W. NIH Image to ImageJ: 25 years of image analysis. Nat. Methods 9, 671 (2012).

58. Rasband, W. Image): Image processing and analysis in Java. (Astrophysics Source Code Library, 1, 2012). 